\title{
TIME MINIMAL SYNTHESIS \\ WITH TARGET OF CODIMENSION ONE UNDER GENERIC CONDITIONS
}

\author{
B. BONNARD and M. PELLETIER \\ Département de Mathématiques, Laboratoire de Topologie, Université de Bourgogne \\ BP 138, 21004 Dijon Cedex, France
}

\begin{abstract}
We consider the problem of constructing the optimal closed loop control in the time minimal control problem, with terminal constraint belonging to a manifold of codimension one, for systems of the form $\dot{v}=X+u Y,|u| \leq 1$ and $v \in \mathbf{R}^{2}$ or $\mathbf{R}^{3}$, under generic assumptions. The analysis is localized near the terminal manifold and is developed to control a class of chemical systems.
\end{abstract}

1. Introduction. Consider a system of the form

$$
\dot{v}=X(v)+u Y(v), \quad v \in \mathbf{R}^{n},
$$

where $X$ and $Y$ are analytic vector fields and admissible controls are measurable scalar functions with values in $[-1,+1]$. Let $N$ be a regular analytic submanifold of $\mathbf{R}^{n}$ of codimension one. We study the following local problem. Let $v_{0} \in N$, compute in a sufficiently small neighborhood $U$ of $v_{0}$ the optimal closed loop function for the time minimal control problem where the target is $N$ and system (1) is restricted to $U$. This analysis is motivated by the control of chemical batch reactors, see [3]. Our aim is to classify all the syntheses in terms of inequalities between the coefficients of the analytic expansions of $X, Y$ and $f$, where $N$ is locally the image of $f$. The generic classification is presented for the planar case in [4]. The aim of this article is to complete this classification in the 3-dimensional case. We use normal forms in order to evaluate the switching and cut loci. We restrict our analysis to the generic situations and describe the topological features of the syntheses.

2. Preliminaries. Consider system (1) written as $(X, Y)$. Let $\hat{N}=\{(v, p)$; $\left.\langle p, w\rangle=0 \forall w \in T_{v} N\right\}$, where $\langle$,$\rangle denotes the standard inner product. An extremal$

1991 Mathematics Subject Classification: 49B10, 93C10.

The paper is in final form and no version of it will be published elsewhere. 
$(v, u)$ is a trajectory of $(1)$, defined on $[T, 0] T<0$ and solution of the maximum principle for the time minimum problem, when the terminal manifold is reduced to a point (This problem is called the point to point problem):

$$
\dot{v}=\frac{\partial H}{\partial p}, \quad \dot{p}=-\frac{\partial H}{\partial v}, \quad H(v, p, u)=\operatorname{Max}_{|w| \leq 1} H(v, p, w)
$$

where $H(v, p, w)=\langle p, X+w Y\rangle$ is the Hamiltonian and $t \rightarrow \operatorname{Max}_{|w|<1} H(v, p, w)$ is a positive constant. A triplet $(v, p, u)$ is called an extremal lift. It will be called a $B C$-extremal lift if it satisfies the boundaries conditions $(v(0), p(0)) \in \hat{N}$ (transversality condition). Let $(v, u)$ be an extremal on $[T, 0]$; a time $s \in[T, 0]$ is called a switching time if $s$ belongs to the closure of the set of $t$ 's in $[T, 0]$ where $v$ is not $C^{1}$ and $v(s)$ will be called a switching point. We shall denote by $W$ the set of switching points for BC-extremals and $K$ the switching locus of optimal trajectories. Let $(v, u)$ be a BC-extremal defined on $[T, 0]$. We call cut point along $v(t)$ the first point where the extremal ceases to be optimal. We denote by $C$ the cut locus. Our aim is to stratify $K \cup C$ and to compute the optimal control as a feedback $v \longmapsto u^{*}(v)$.

Let $v \in \mathbf{R}^{n}$ and let $\mathcal{C}(v)$ be the convex set $\{X(v)+u Y(v) ;|u| \leq 1\}$. Let $v_{0} \in N$, in our analysis we shall assume that $Y\left(v_{0}\right)$ is not zero, moreover it is not restrictive to suppose that $\mathcal{C}\left(v_{0}\right)$ lies entirely in one half-space limited by $T_{v_{0}} N$. Let $n(v)$ be the unit normal to $N$ oriented near $v_{0}$ as $\mathcal{C}\left(v_{0}\right)$.

3. Classification. We classify by increasing the codimension of the singularities in the jet-space of $(X, Y, f)$ at $\left(v_{0}, f^{-1}\left(v_{0}\right)\right)$. We present the generic classification when $v \in \mathbf{R}^{2}$ or $\mathbf{R}^{3}$. Due to space restrictions, the proofs are only sketched but we illustrate the techniques used to handle the problem.

3.1. Generic case. Let us assume that both $X\left(v_{0}\right) \pm Y\left(v_{0}\right)$ are not tangent to $N$. Let $n\left(v_{0}\right)$ be the normal to $N$ oriented with the convention $\left\langle n\left(v_{0}\right), X\left(v_{0}\right) \pm\right.$ $\left.Y\left(v_{0}\right)\right\rangle>0$. Let $(v, p, u)$ be a BC-extremal defined on $[T, 0]$. From the maximum principle one can set $p(0)=n(v(0))$, for $\left|v(0)-v_{0}\right|$ small. Let us assume $\left\langle n\left(v_{0}\right), Y\left(v_{0}\right)\right\rangle \neq 0$, then from the transversality condition, the optimal synthesis in a sufficiently small neighborhood of $v_{0}$ is given in Fig. 1,
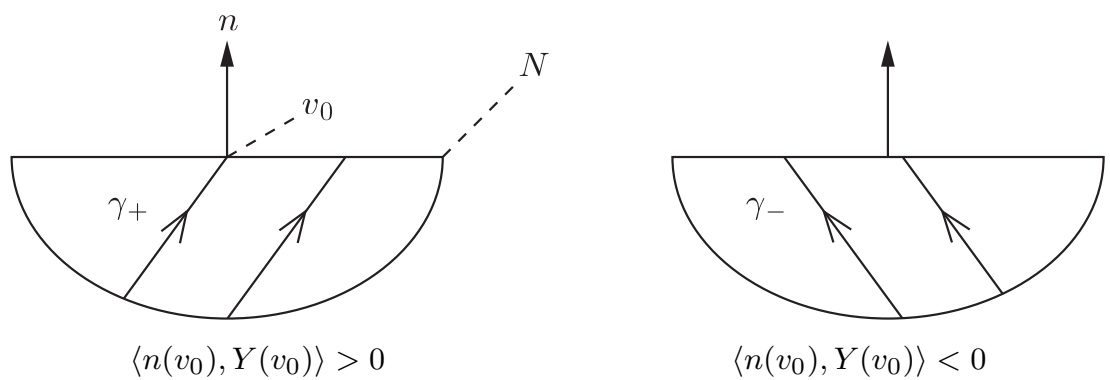

Fig. 1 
where $\gamma_{+}$(resp. $\gamma_{-}$) are arcs corresponding to $u=+1$ (resp. $u=-1$ ).

3.2. Generic switching point. Let $v_{0} \in N$ such that $\left\langle n\left(v_{0}\right), Y\left(v_{0}\right)\right\rangle=0$, where $n$ is the normal to $N$. In order to analyze this singularity one need preliminary lemmas.

3.2.1. Lemma. Let $v_{0} \in N$ be such that $\left\langle n\left(v_{0}\right), Y\left(v_{0}\right)\right\rangle=0$ and let us assume $\left\langle n\left(v_{0}\right),[X, Y]\left(v_{0}\right)\right\rangle \neq 0$, the Lie bracket of two vector fields being computed with the convention

$$
\left[Z_{1}, Z_{2}\right](v)=\frac{\partial Z_{2}}{\partial v}(v) Z_{1}(v)-\frac{\partial Z_{1}}{\partial v}(v) Z_{2}(v) .
$$

Then $(v, p, u \equiv 1)$ (resp. $(v, p, u \equiv-1))$ is a BC-extremal lift with $v(0)=v_{0}$, $p(0)=n\left(v_{0}\right)$ if and only if $\left\langle n\left(v_{0}\right),[X, Y]\left(v_{0}\right)\right\rangle<0$ (resp. $\left.>0\right)$.

Proof. We prove the assertion when $u \equiv 1$. Assume $(v, p, u \equiv 1)$ is a $B C$-extremal lift defined on $[T, 0]$ with $v(0)=v_{0}, p(0)=n\left(v_{0}\right)$. Let $\Phi^{+}$be the switching function $\langle p, Y(v)\rangle$ evaluated along the extremal. We have $\Phi^{+}(t)=$ $\Phi^{+}(0)+t \dot{\Phi}^{+}(0)+o(t)$ and computing we get $\dot{\Phi}^{+}=\langle p,[X, Y](v)\rangle$. Since $\Phi^{+}(0)=0$ and $u(t)=\operatorname{sign} \Phi^{+}(t)=+1$ for $t<0$, we must have $\left\langle p(0),[X, Y]\left(v_{0}\right)\right\rangle<0$.

3.2.2. Lemma. Let us assume $\left\langle n\left(v_{0}\right),[X, Y]\left(v_{0}\right)\right\rangle \neq 0$. Then, the arcs $\gamma_{+}$and $\gamma_{-}$arriving at $v_{0}$ cannot be sets of input switching points.

Proof. For instance, let us assume that $(v, p, u \equiv 1)$ is a $B C$-extremal on $[T, 0]$ with $v(0)=v_{0}$, then $p(0) \in \mathbf{R} n\left(v_{0}\right)$. Moreover assume that each point of $v$ is an input switching point. Hence there exists $B C$-extremals $\gamma=\gamma_{-} \gamma_{+}$, where $\gamma_{+}$is any subarc of $v(t)$ and $\gamma_{-} \gamma_{+}$designs an arc $\gamma_{-}$followed by an arc $\gamma_{+}$. Then we have $\langle p(t), Y(v(t))\rangle=0, \forall t \in[T, 0]$. Differentiating with respect to $t$ and evaluating at $t=0$, we get $\left\langle p(0),[X, Y]\left(v_{0}\right)\right\rangle=0$. This is absurd since $p(0) \in \mathbf{R} n\left(v_{0}\right)$.

3.2.3. Assumptions. From now on, we assume $\left\langle n\left(v_{0}\right), Y\left(v_{0}\right)\right\rangle=0$ and both $\left\langle n\left(v_{0}\right), X\left(v_{0}\right)\right\rangle$ and $\left\langle n\left(v_{0}\right),[X, Y]\left(v_{0}\right)\right\rangle$ non zero, $n\left(v_{0}\right)$ being oriented with the convention $\left\langle n\left(v_{0}\right), X\left(v_{0}\right)\right\rangle>0$. Moreover, we suppose $v=(x, y) \in \mathbf{R}^{2}$, the generalization being straightforward.

3.2.4. Method of analysis. In order to evaluate near $v_{0}$ the switching locus $K$ and the cut locus $C$ it is convenient to make the following normalizations.

First, one may set $v_{0}=(0,0)$ and as in [2], since $X$ and $Y$ are transverse at $v_{0}$, one may assume locally $Y=\frac{\partial}{\partial y}$ and that the trajectory corresponding to $u \equiv 0$ is $t \rightarrow(t, 0)$. Hence $(1)$ can be written locally:

$$
\dot{x}=1+\sum_{i=1}^{+\infty} a_{i}(x) y^{i}, \quad \dot{y}=\sum_{i=1}^{+\infty} b_{i}(x) y^{i}+u .
$$

Moreover, changing if necessary $y$ into $-y$ and $u$ into $-u$, one can assume $a=$ $a_{1}(0)>0$ where $a=-\langle n(0),[X, Y](0)\rangle, n(0)=(1,0)$ being the unit normal to $N$. Since $Y$ is tangent to $N$ at 0 , the terminal manifold can be locally parametrized by 
$s \mapsto(c(s), s)$, where $c(s)=k s^{2}+o\left(s^{2}\right)$ and $k$ represents the curvature of $N$ which is generically $\neq 0$. We have $n(0)=(1,0)$ and for $v$ small, using $\langle n(v), X(v)\rangle>0$ one can set $n(v)=\left(n_{1}, n_{2}\right), n_{1}=1, n_{2}=-c^{\prime}(s)=-2 k s+o(s)$. Hence, for $s$ small we have: if $k<0$, then $n_{2}>0$ if $s>0$ and $n_{2}<0$ if $s<0$, and conversely if $k>0$. The Hamiltonian is $H(v, p, u)=\langle p, X+u Y\rangle$ and if $v \in N$ is small, $p=n(v)$, its maximum over $|u| \leq 1$ is obtained as follows: if $k<0, s>0$, then $n_{2}>0$ and $u$ maximizing $H$ is +1 if $s<0$, it is -1 and conversely if $k>0$. Hence, we get the following important geometric behaviors: if $k<0$, the arcs $\gamma_{+}$ and $\gamma_{-}$satisfying the transversality conditions can cut themselves, contrarily to the case $k>0$.
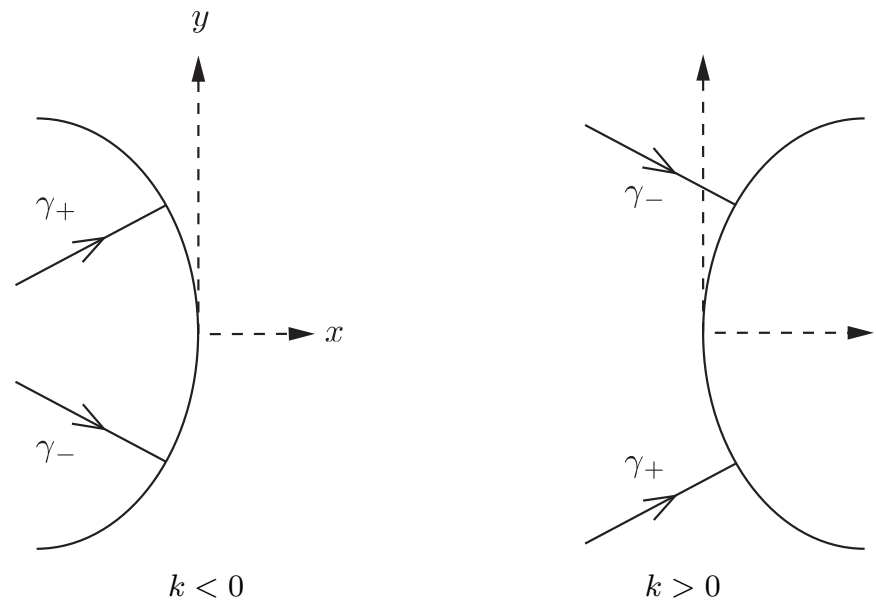

$k>0$

Fig. 2

The adjoint system associated to $(2)$ with $p=\left(p_{1}, p_{2}\right)$ is

$$
\begin{aligned}
& \dot{p}_{1}=-p_{1} \sum_{i=1}^{+\infty} a_{i}^{\prime}(x) y^{i}-p_{2} \sum_{i=1}^{+\infty} b_{i}^{\prime}(x) y^{i}, \\
& \dot{p}_{2}=-p_{1} \sum_{i=1}^{+\infty} i a_{i}(x) y^{i-1}-p_{2} \sum_{i=1}^{+\infty} i b_{i}(x) y^{i-1},
\end{aligned}
$$

where $a_{i}^{\prime}$ and $b_{i}^{\prime}$ are the derivatives of $a_{i}$ and $b_{i}$ with respect to $x$. If $u$ is a piecewise analytic control every solution $(v, p)$ of $(2),(3)$ such that $(v(0), p(0)) \in \hat{N}$, can be evaluated for $t$ sufficiently small, by analyticity.

3.2.5. Lemma. Near 0, every optimal solution is of the form $\gamma_{+} \gamma_{-}$.

Proof. From [5], we know that every $B C$-extremal is of this form and from [9], we know that every optimal solution for the point to point problem is of this form. Now let $v_{1}, v_{2}$ be two points near 0 and let $\gamma_{1}=\gamma_{+} \gamma_{-}$and $\gamma_{2}=\gamma_{-} \gamma_{+}$be two arcs defined on $\left[0, t_{1}\right]$ and $\left[0, t_{2}\right]$ joining $v_{1}$ to $v_{2}$. To compare $t_{1}$ and $t_{2}$ we introduce the one form $\omega$ defined by $\omega(Y)=0$ and $\omega(X)=1$. From Stokes' theorem we 
have:

$$
\int_{\gamma_{1}} \omega-\int_{\gamma_{2}} \omega=t_{1}-t_{2}=\int_{D} d \omega
$$

where $D$ is the domain limited by $\gamma_{1} \vee \gamma_{2}$. Computing with $d \omega>0$ and using the fact that $\gamma_{1} \vee-\gamma_{2}$ is clockwise we have $t_{1}<t_{2}$.

3.2.6. Lemma. The arc $\gamma_{-}$arriving at 0 is not optimal.

Proof. $n(0)=(1,0)$ and computing we have: $\langle n(0),[X, Y](0)\rangle=-a<0$. Hence from lemma 3.2.1, this arc is not a $B C$-extremal.

3.2.7. Lemma. Assume $k \neq 0$, then the switching points of $B C$-extremals $\gamma_{+} \gamma_{-}$near 0 form an analytic curve $W$ starting from 0 and whose tangent at 0 is $\mathbf{R}(-2 k / a, 1+2 k / a)$.

P r o of. We integrate $(2),(3)$ backwards in time, with initial conditions $v(0) \in$ $N, p(0)=n(v(0))=(1,-2 k s+o(s))$. We get $p_{1}(t)=1+o(1), p_{2}(t)=-2 k s-a t+$ $o(|s, t|)$. If $k<0$, we must have $s<0$ and if $k>0, s>0$. Hence the $B C$-extremal $\gamma_{-}$is switching at $(x(w), y(w))=s(-2 k / a, 1+2 k / a)+o(s)$.

3.2.8. Lemma. Near 0 a BC-extremal $\gamma_{+} \gamma_{-}$is crossing $W$ if $k>0$ or $-a / 4<$ $k<0$ and is reflecting on $W$ if $k<-a / 4$.

Proof. We compare the respective slopes of $W, \gamma_{+}$and $\gamma_{-}$at 0 . They are $-1-a / 2 k,+1$ and -1 . Hence if $k>0$, the slope of $W$ is less than -1 . If $k<0$, $-1-a / 2 k>1$ if and only if $-a / 4<k$. Hence the geometric situations are:

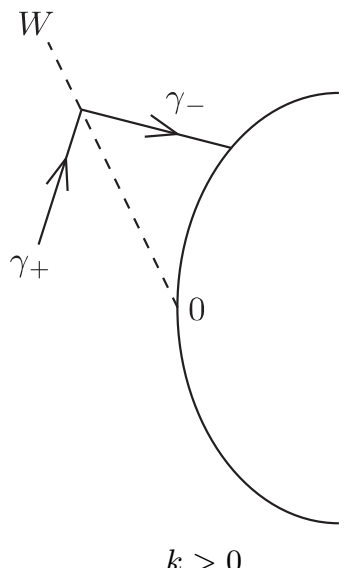

$k>0$

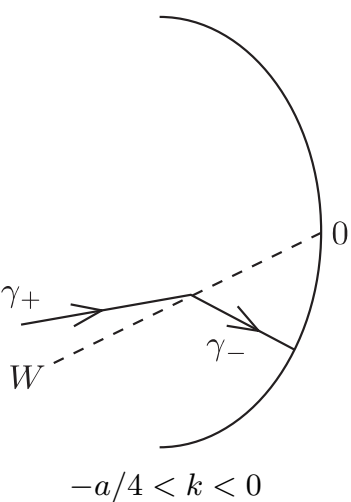

Fig. 3

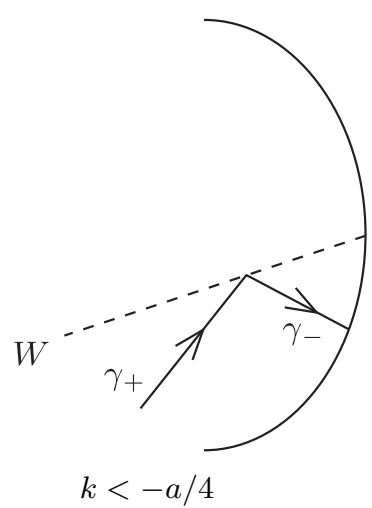

$k<-a / 4$

3.2.9. Proposition. The optimal syntheses are given in Fig. 4, where $K$ is an analytic curve with slope at $0,-1-a / 2 k$ and in the third case the cut locus $C$ is an analytic curve with slope at 0 equal to $-a / 4 k$. 


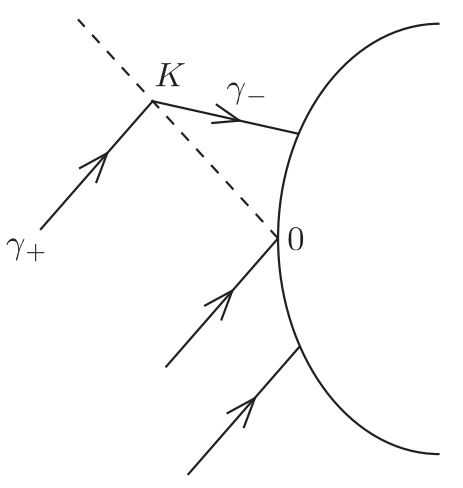

$k>0$

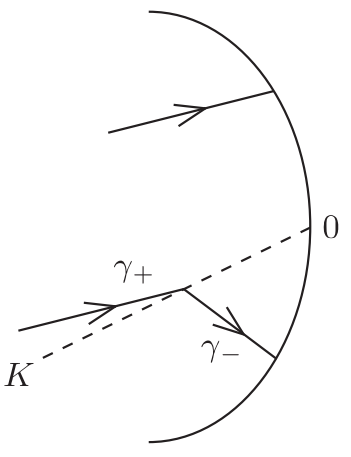

$-a / 4<k<0$

Fig. 4

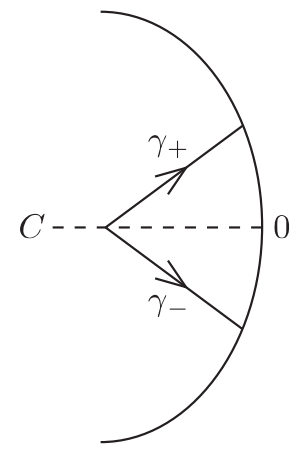

$k<-a / 4$

P r o of. In the first two cases, the situation is clear because from each point near 0 , to the left of $N$, there exists only one $B C$-extremal $\gamma_{+} \gamma_{-}$. In the third case, more that one $B C$-extremal $\gamma_{+} \gamma_{-}$is possible to reach the target. Let us prove than an optimal policy has no switching. Assume that optimal trajectories $\gamma_{+} \gamma_{-}$accumulate near 0 (Fig. 5.1). Then we can construct optimal trajectories of the form $\gamma_{+} \gamma_{-} \gamma_{+} \gamma_{-}$which is absurd. The cut locus is constructed as follows. Let $L$ be subanalytic set $\{v=(x, y), v$ small, $x<0$, such that both $\exp t(X \pm Y)(v)$ intersect $N\}$ where $\exp t Z$ is the local parameter group associated to the vector field $Z$. By computing we get that $L$ is an analytic curve whose tangent at 0 is $-a / 4 k \in] 0,1]$ (Fig. 5.2). Clearly $C=L$.

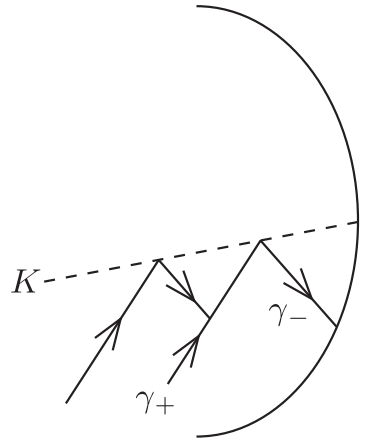

Fig. 5.1

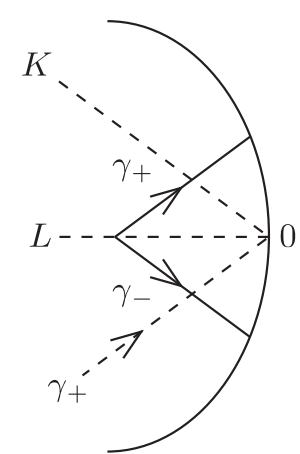

Fig 5.2

4. Generic fold case. In this section we analyse the situation when a singular extremal meets the terminal manifold at a point where $Y$ and $[X, Y]$ are tangent to $N$. It is a generic situation when $v \in \mathbf{R}^{n}, n \geq 3$. We shall restrict our analysis to the case $n=3$.

4.1. Preliminaries. Consider system (1) where $v=(x, y, z) \in \mathbf{R}^{3}$. An extremal lift $(v, p, u)$ is called singular on $[0, T]$ if $\langle p(t), v(t)\rangle=0 \forall t \in[0, T]$. Let $D=$ $\operatorname{det}(Y,[X, Y],[Y,[X, Y]]), D^{\prime}=\operatorname{det}(Y,[X, Y],[X,[X, Y]])$ and $S=\{v ; D(v)=0\}$. 
4.1.1. Lemma. The singular extremals $(v, u)$ contained in $\mathbf{R}^{3} \backslash S$ are the solutions of

$$
\dot{v}=X(v)+\hat{u}(v) Y
$$

where $u(t)=\hat{u}(v(t))=-D^{\prime} / D$.

Proof. By differentiating twice the equation $\langle p(t), Y(v(t))\rangle=0, t \in[0, T]$ one gets $\langle p(t),[X, Y](v(t))\rangle=\langle p(t),[X,[X, Y]](v(t))+u(t)[Y,[X, Y]](v(t))\rangle=0$. Since $p \neq 0$ this implies $D^{\prime}+u D=0$ and $u$ can be computed on $\mathbf{R}^{3} \backslash S$ as a feedback $\hat{u}$.

4.1.2. Definition. Let $(v, p, u)$ be a singular extremal lift on $[0, T]$. We assume the following.

H0. $t \rightarrow v(t)$ is a one to one mapping contained in $\mathbf{R}^{3} \backslash S$ (Hence analytic).

$H 1$. For each $t \in[0, T], Y(v(t))$ and $[X, Y](v(t))$ are linearly independent. The adjoint vector $p$ is oriented with the convention $h=H(v(t), p(t), u(t))=$ $\langle p(t), X(v(t))\rangle \geq 0$. The extremal is called hyperbolic if $\forall t \in[0, T], h \neq 0$ and $\left\langle p(t), a d^{2} Y(X)(v(t))\right\rangle<0$, elliptic if $h \neq 0$ and $\left\langle p(t), a d^{2} Y(X)(v(t))\right\rangle>0$ and exceptional if $h=0$.

From [2] we have

4.1.3. Proposition. Assume $T>0$ enough small. Then $(v, u)$ defined on $[0, T]$ is time minimizing (resp. maximizing) with respect to all solutions of $\dot{v}=$ $X+u Y, u \in \mathbf{R}$, joining $v(0)$ to $v(T)$ and contained in a sufficiently small neighborhood of $v$ if it is hyperbolic or exceptional (resp. elliptic).

4.2. Normalizations. Let $v_{0} \in N$ such that $Y\left(v_{0}\right)$ and $[X, Y]\left(v_{0}\right)$ are tangent to $N$. We assume that $v_{0}=0$ and $N$ is the image by an immersion $f: q \in \mathbf{R}^{2} \mapsto \mathbf{R}^{3}$ of a neighborhood $U$ of 0 . We shall normalize under generic assumptions (1) and $N$, near 0 , using the action of the pseudo-group $G$ generated by the following transformations:

(i) Local diffeomorphism $\psi: \mathbf{R}^{2} \mapsto \mathbf{R}^{2}, \psi(0)=0$ changing the parametrization $q$ of $N$.

(ii) Local diffeomorphism $\varphi: \mathbf{R}^{3} \longrightarrow \mathbf{R}^{3}, \varphi(0)=0$ of the state space.

(iii) Feedback transformation $u \mapsto-u$ (the trajectories $\gamma_{+}$and $\gamma_{-}$are interchanged).

4.2.1. Proposition. Under generic assumptions, and using the action of $G$, system (1) can be written near 0 as

$$
\begin{aligned}
& \dot{x}=1+a z^{2}+a^{\prime} y z+a^{\prime \prime} y^{2}+R_{1}, \\
& \dot{y}=b z+b^{\prime} y+R_{2}, \\
& \dot{z}=(u-\hat{u}(0))+c x+c^{\prime} y+c^{\prime \prime} z+R_{3}
\end{aligned}
$$


where $a \neq 0, a^{\prime}>0, b \neq 0, \hat{u}(0) \geq 0$ and $R_{1}=o\left(|x, y, z|^{3}\right), R_{2}, R_{3}=o\left(|x, y, z|^{2}\right)$. The singular extremal passing through 0 being indentified to $t \mapsto(t, 0,0)$ and $N$ is the image of $f$ where $f(s, w)=\left(k s^{2}, w, s\right)+o\left(|s, w|^{2}\right)$.

Proof. We sketch the proof indicating the geometric interpretation of this result.

First, if $Y(0) \neq 0$, one may set locally $Y=\frac{\partial}{\partial z}$. We assume $0 \in \mathbf{R}^{3} \backslash S$ and that the singular extremal passing through 0 is one to one. Using a diffeomorphism leaving $Y=\frac{\partial}{\partial z}$ invariant, it can be transformed into $t \mapsto(t, 0,0)$.

Now let $d$ be the set of points where $Y$ is tangent to $N$. Assume $d$ is a simple curve transverse to $0 z$ and $0 x$. Hence using a local diffeomorphism of $\mathbf{R}^{3}$ preserving $Y=\frac{\partial}{\partial z}$ and each point of the axis $0 x$, we may apply $d$ onto the axis $0 y$.

Now, at 0 , the tangent space to $N$ is $\mathbf{R}\left\{\frac{\partial}{\partial y}, \frac{\partial}{\partial z}\right\}$. By assumption $[X, Y]$ is tangent to $N$ at 0 and near $0 \in S$, the two vector fields $Y$ and $[X, Y]$ are linearly independent. By computing, one see that there exists a diffeomorphism preserving the previous normalizations and transforming $[X, Y]$ along $0 x$ into the constant vector $[X, Y](0)$.

Hence we get (5). The singular extremal identified to $t \longrightarrow(t, 0,0)$ is hyperbolic (resp. elliptic) if and only if $a<0$ (resp. $a>0$ ). The condition $a^{\prime} \neq 0$ means that the set $d^{\prime}$ where $[X, Y]$ is tangent to $N$ is a simple curve transverse to $0 y$, which corresponds to the set of points where $Y$ is tangent to $N$. The conditions $a^{\prime}>0$ and $\hat{u}(0) \geq 0$ are obtained by changing if necessary $y$ into $-y, z$ into $-z$ and $u$ into $-u$.

Now we can normalize $f$ using changes of parametrization. Since at 0 , the tangent space to $N$ is $\mathbf{R}\left\{\frac{\partial}{\partial y}, \frac{\partial}{\partial z}\right\}$, from the implicit function theorem one may assume $f(s, w)=(Q(s, w), w, s)+o\left(|s, w|^{2}\right)$ where $Q$ is a quadratic form. By construction $N$ contains the axis $0 y$ and at each point of this line its tangent space is $\mathbf{R}\left\{\frac{\partial}{\partial y}, \frac{\partial}{\partial z}\right\}$. This implies $Q(s, w)=k s^{2}$.

The proposition is then proved.

4.3. Notations. We denote $\Gamma_{\varepsilon}$ the union of the trajectories corresponding to the constant control $u=\varepsilon, \varepsilon= \pm 1$, reaching $N$ at $(0, w, 0)$. Let $(v, p, u)$ be an extremal defined on $[0, T], T \in \mathbf{R}$, with $v(0) \in N, p(0)=n(v)$ and let $p=\left(p_{1}, p_{2}, p_{3}\right)$. Let $t_{1}$ be the first $|t|$ such that $p_{3}(t)=0$. Hence on $\left[0, t_{1}\right], u=\varepsilon, \varepsilon= \pm 1$ and we shall denote $K_{\varepsilon}$ the union of the points $v\left(t_{1}\right)$. By construction $K_{\varepsilon}$ contains the locus of first switching points of $B C$-extremals.

Using model (5) we shall evaluate $\Gamma_{\varepsilon}$ and $K_{\varepsilon}$.

4.4. LemMA. The set $\Gamma_{\varepsilon}$ is given by the equations: $x=t+o\left(|w, t|^{2}\right) y=w+$ $b(\varepsilon-\hat{u}) t^{2} / 2+b^{\prime} w t+o\left(|w, t|^{2}\right), z=(\varepsilon-\hat{u}) t+c t^{2} / 2+c^{\prime} w t+c^{\prime \prime}(\varepsilon-\hat{u}) t^{2} / 2+o\left(|w, t|^{2}\right)$. Near 0 , it is a 2-dimensional analytic manifold whose tangent space is given by $z=\left[(\varepsilon-\hat{u})+c^{\prime} w\right] x+o\left(w^{2}\right)$ at $(0, w, 0)$.

Pro of. Computations. 
4.5. Lemma. The time $t_{1}$ is given by solving:

$$
p_{3}(t)=-2 k s-a t^{2}(\varepsilon-\hat{u})-a^{\prime} w t+o\left(|s, w, t|^{3}\right)=0
$$

where the weight of the variables is 1 for $t, w$ and 2 for $s$, if $k \neq 0$. The set $K_{\varepsilon}$ is parametrized by $x=t_{1}+o\left(\left|t_{1}, w\right|^{2}\right), y=w+b(\varepsilon-\hat{u}) t_{1}^{2} / 2+b^{\prime} w t_{1}+o\left(\left|t_{1}, w\right|^{2}\right)$, $z=(\varepsilon-\hat{u}) t_{1}+\left[c+(\varepsilon-\hat{u})\left(c^{\prime \prime}-a / k\right)\right] t_{1}^{2} / 2+\left(c^{\prime}-a / 2 k\right) w t_{1}+o\left(\left|t_{1}, w\right|^{2}\right)$. Near 0 , it is an analytic manifold whose tangent space is given by $z=\left[(\varepsilon-\hat{u})+\left(c^{\prime}-\right.\right.$ $a / 2 k) w] x+o\left(w^{2}\right)$ at $(0, w, 0)$.

Pro of. Computations.

4.6. R e mark. We observe that at 0 both $\Gamma_{\varepsilon}$ and $K_{\varepsilon}$ are tangent. The synthesis depends of the relative position of $\Gamma_{\varepsilon}$ and $K_{\varepsilon}$ near 0 . The easy case is the case $k<0$.

4.7. Proposition. If $k<0$, the optimal trajectories are of the form $\gamma_{+}$or $\gamma_{-}$and the synthesis is described in Fig. 6, in each plane $y=c, c$ small.

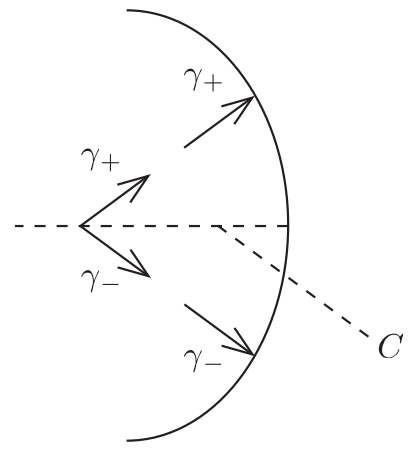

Fig. 6

Proof. In this case the $B C$-extremals are cutting themselves before to be allowed to switch. Hence, there exists a cut locus (subanalytic set) whose intersection with a plane $y=c$ is homeomorphic to a line.

When $k>0$, the situation is intricate. One must distinguish different cases: $a>0, a<0,1>\hat{u}(0) \geq 0, \hat{u}(0)=1$ (saturating singular control), $\hat{u}(0)>1$ (not admissible).

4.8. Proposition. Assume $k>0, a<0$ and $1>\hat{u}(0) \geq 0$. Then near 0 , the optimal synthesis is homeomorphic to that of Fig. 7 , where $\gamma_{s}$ designs a singular arc. 


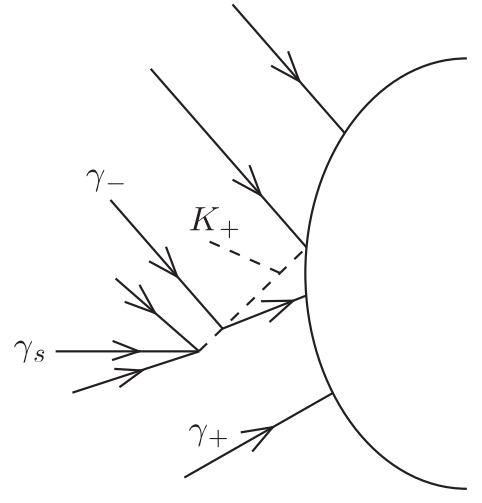

$y<0$

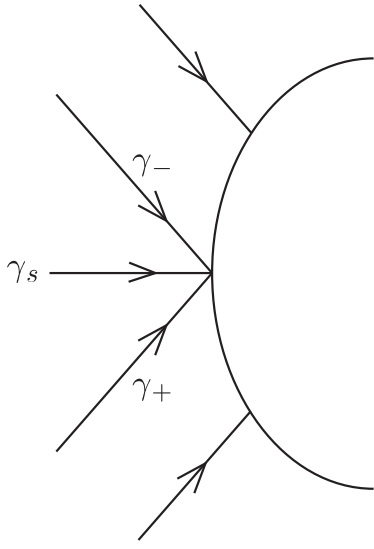

$y=0$

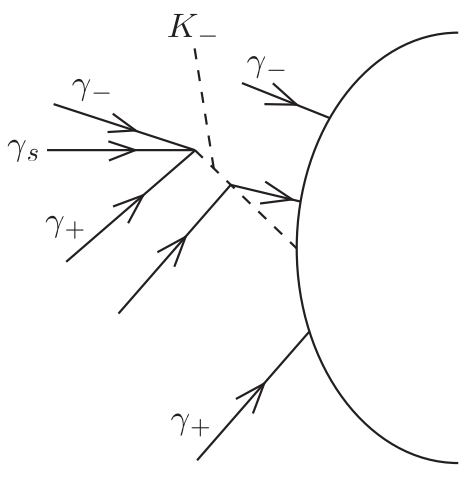

$y>0$

Fig. 7

Proof. Let $m_{0}=(0, n(0))$, where $n(0)=(1,0,0)$ (normal to $\left.N\right)$. Now using the classification from [5], $m_{0}$ is an hyperbolic fold point and near $m_{0}$ the behavior of BC-extremal lifts is as shown in Fig. 8, where $m_{0}$ belongs to $\langle p, Y\rangle=\langle p,[X, Y]\rangle=0$ and the singular extremal lifts are contained in this surface. This classification is sufficient to compute the optimal synthesis.

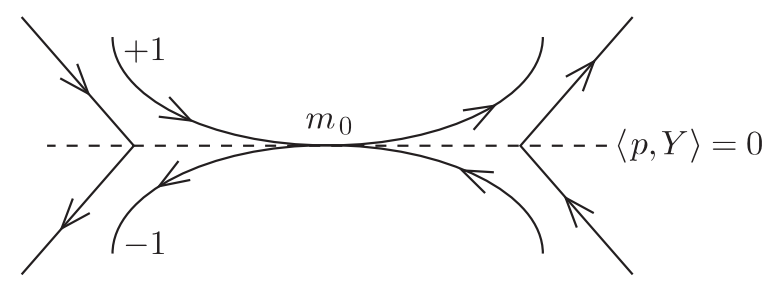

Fig. 8

Indeed, we stratify $N$ using the transversality condition and along $0 y$, lemma 3.2.1, we have the situation of Fig. 9, and from Fig. 8, at 0, a $B C$-extremal law can be $+1,-1$ or $\hat{u}(0)$.

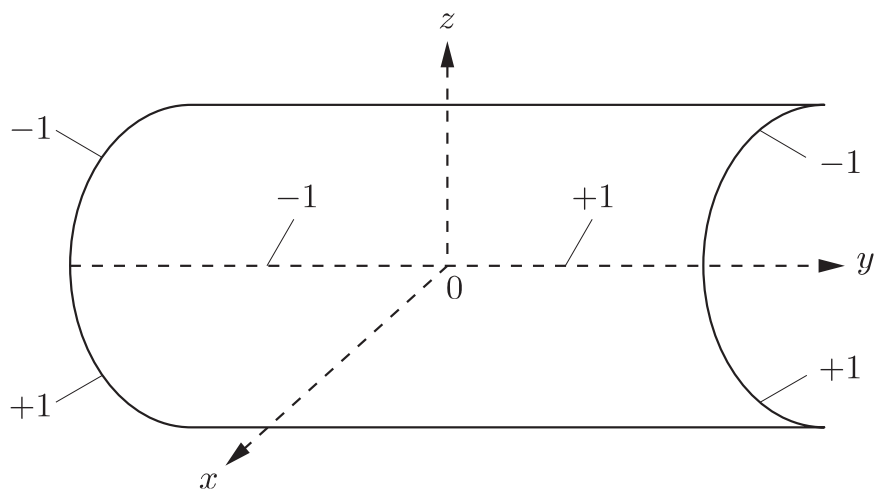

Fig. 9 
Now, we compute the set of first switching points for $B C$-extremals. By definition they are located on manifolds $K_{\varepsilon}$ defined by lemma 4.5. A switching time $t_{1}$ is approximated by solving:

$$
a(\varepsilon-\hat{u}) t^{2}+a^{\prime} w t+2 k s=0 .
$$

If $\delta=a^{\prime 2} w^{2}-8 k a(\varepsilon-\hat{u}) s$, we must have $\delta \geq 0$ and

$$
t_{1}=\frac{-a^{\prime} w \pm \sqrt{\delta}}{2 a(\varepsilon-\hat{u})} \leq 0 .
$$

Moreover from the transversality condition we must have $\varepsilon s \leq 0$ (Fig. 9). Computing and taking the smallest $\left|t_{1}\right|$, which $\longrightarrow 0$ when $s \rightarrow 0$, for each $w$, we get the set of first switchings points shown in Fig. 10.

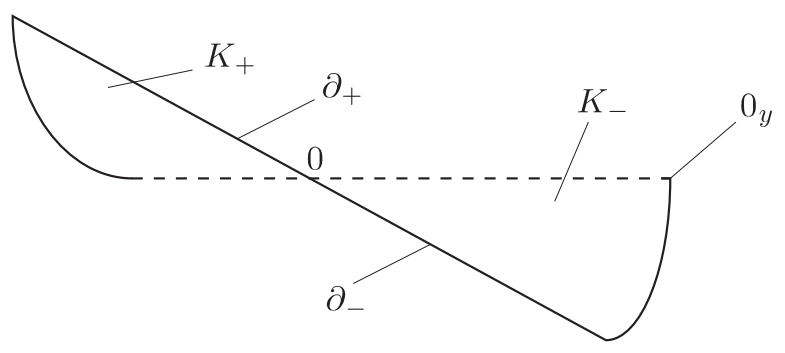

Fig. 10

At a switching point such that $\langle p,[X, Y]\rangle=0$, an extremal control can be +1 , -1 or singular. Such points form the boundaries $\partial_{+}$of $K_{+}$and $\partial_{-}$of $K_{-}$.

If we flatten everything by homeomorphism we get the synthesis from Fig. 7 . In this case the optimal synthesis is the unique extremal synthesis and we have represented in Fig. 11 the correspondence between extremal lift and optimal trajectories, in each plane $y=$ constant $>0$.
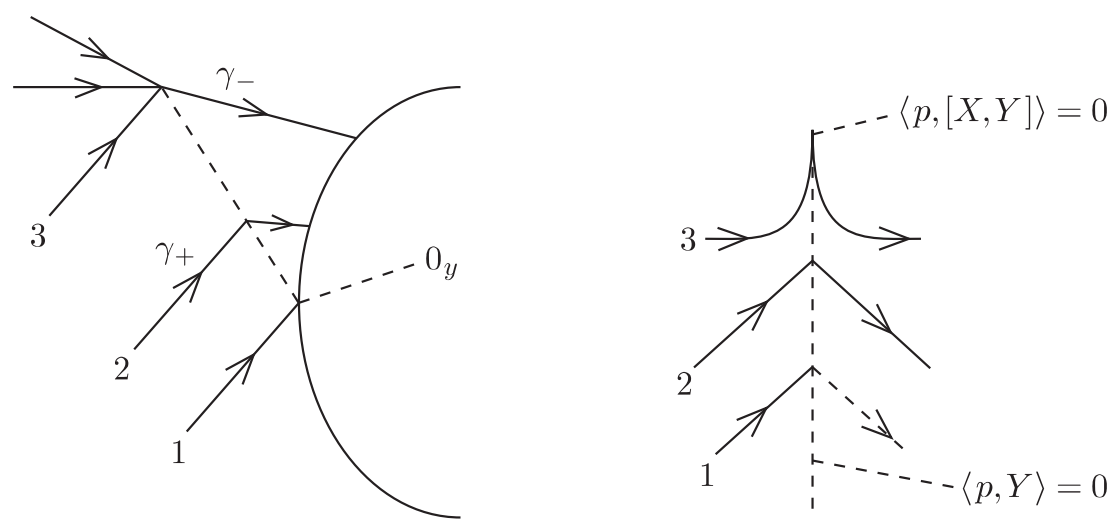

Fig. 11

4.9. Proposition. Assume $k>0, a>0$ and $1>\hat{u}(0) \geq 0$. Then the optimal trajectories have at most one switching and the optimal synthesis is given in each 
plane $y=c$ by Fig. 12, where $K_{+}$(resp. $\left.K_{-}\right)$is the set of switching points corresponding to $\gamma_{-} \gamma_{+}\left(\right.$resp. $\left.\gamma_{+} \gamma_{-}\right)$and $C$ is the cut locus where an optimal control can be $u=1$ or $u=-1$.
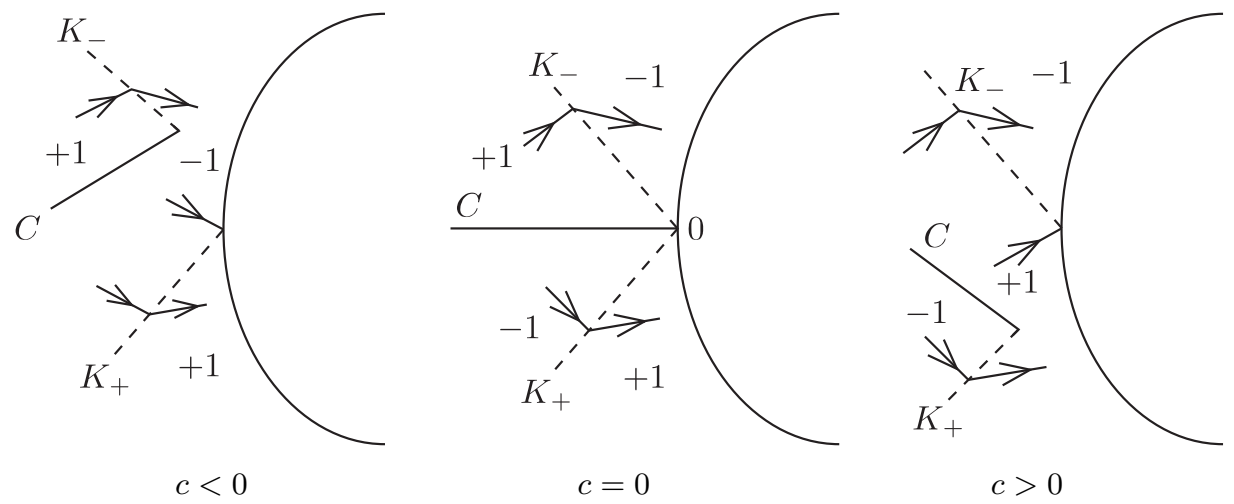

Fig. 12

Sketch of proof. Let $m_{0}=(0, n(0)), n(0)=(1,0,0)$ normal to $N$ at 0 . Since $a>0$, according to [5] the $B C$-extremals lifts near $m_{0}$ are of the form (elliptic case) shown in Fig. 13.

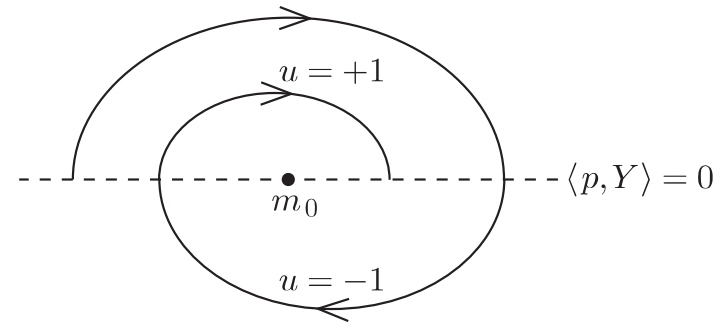

Fig. 13

Here $\langle p, Y\rangle=\langle p,[X, Y]\rangle=0$ contains singular extremal lifts which are elliptic, hence time maximizing, for the point to point time optimal control problem. Moreover a concatenation between a singular $\operatorname{arc} \gamma_{s}$ and an $\operatorname{arc} \gamma_{+}$or $\gamma_{-}$is not extremal and there exists no uniform bounds on the number of switchings of extremals $\gamma_{-} \gamma_{+} \gamma_{-} \gamma_{+} \cdots$. Indeed the number of switchings tends to $+\infty$ when the initial condition for extremals lifts tends to $m_{0}$.

Hence, there exists no BC-extremal passing through 0. This suggests the existence of a cut locus $C \ni 0$.

Now we shall evaluate the set $K_{+}$and $K_{-}$of first switching points.

The stratification of $N$ by $B C$-controls is as before, see Fig. 9 and the switching times are approximated by

$$
t_{1}=\frac{-a^{\prime} w \pm \sqrt{\delta}}{2 a(\varepsilon-\hat{u})}
$$


where $\delta=a^{\prime 2} w^{2}-8 k a(\varepsilon-\hat{u}) s$. Fix $w>0$ and take a point of $N$. For $s>0$, the optimal law is $\varepsilon=+1$ and a $B C$-extremal is switching at

$$
t_{1}=\frac{-a^{\prime} w+\sqrt{\delta}}{2 a(\varepsilon-\hat{u})} .
$$

If $s<0$, the optimal law is $\varepsilon=-1$ and a $B C$-extremal is switching at

$$
t_{1}=\frac{-a^{\prime} w-\sqrt{\delta}}{2 a(\varepsilon-\hat{u})} .
$$

In the first case $t_{1} \rightarrow 0$ when $s \rightarrow 0$ and in the second case $t_{1}$ is of order $w$ when $s \rightarrow 0$. Hence we get the respective sets of switching points shown in Fig. 14, where the distance of $K_{+}$to $N$ is of order $w$.
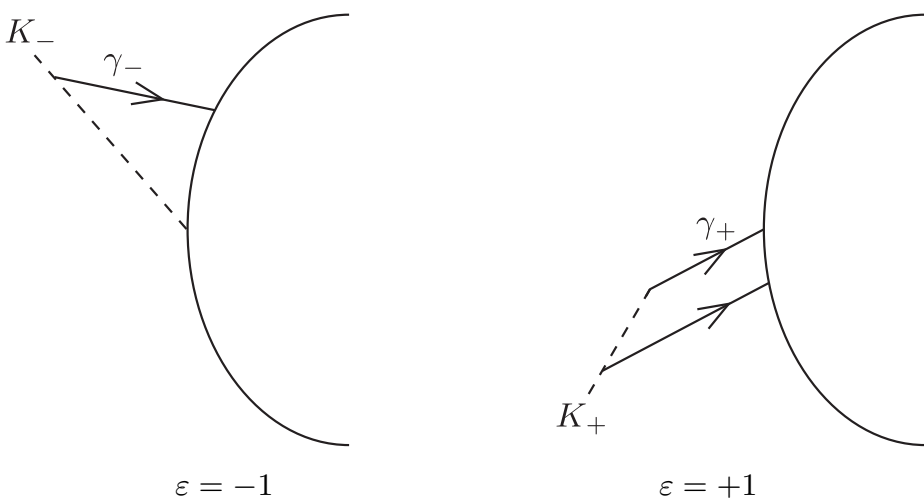

Fig. 14

This phenomenon has the following interpretation. From lemmas 4.4 and 4.5 both manifolds $K_{\varepsilon}$ and $\Gamma_{\varepsilon}$ are tangent at 0 . Now, at $(0, w, 0)$ their respective tangent space are $z=\left[(\varepsilon-\hat{u})+\left(c^{\prime}-a / 2 k\right) w\right] x$ and $z=\left[(\varepsilon-\hat{u})+c^{\prime} w\right] x$. Hence for $w>0$ and $\varepsilon=+1, \Gamma_{\varepsilon}$ is below $K_{\varepsilon}$ and to compute the switching points we have to know the curvature of $K_{\varepsilon}$, see Fig. 15.

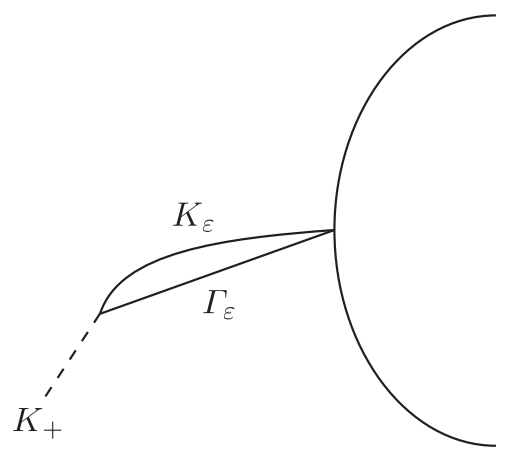

Fig. 15 
The cut locus $C$ is a subanalytic set whose intersections with $y=c$ are described in Fig. 12.

4.10. Proposition. Assume $k>0, \hat{u}(0)>1$. Then if $a>0$, the optimal trajectories have at most two switchings and the optimal synthesis is given in each plane $y=c$ by Fig. 16, where $K_{-}^{2}$ represents the set of second switching points. If $a<0$, the situation is similar.

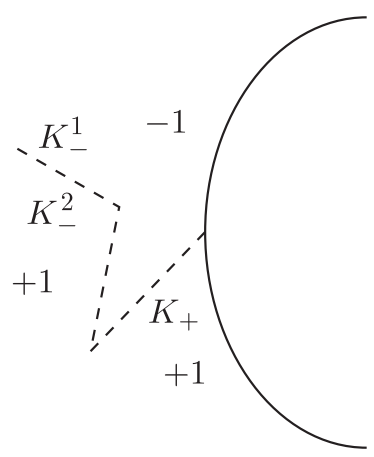

$c<0$

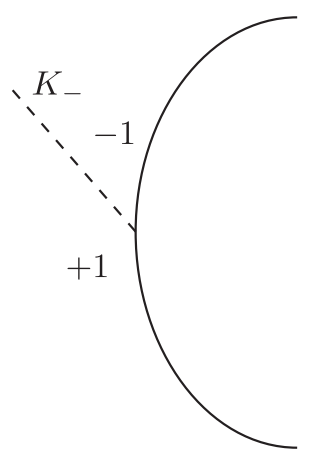

$c=0$

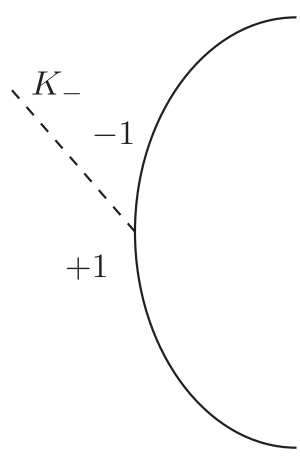

$c>0$

Fig. 16

Sketch of proof. In this case $m_{0}=(0, n(0))$ corresponds in [5] to a parabolic point and near $m_{0}$ the $B C$-extremals lifts are of the form shown in Fig. 17.

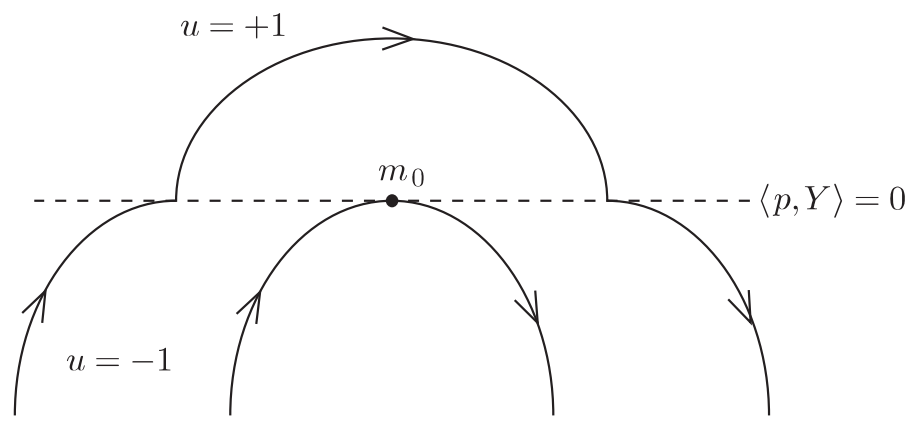

Fig. 17

Hence a $B C$-extremal has near $z_{0}$ at most two switchings. Since $\hat{u}(0)>1$ the singular BC-extremal passing through 0 is not admissible. The synthesis follows by evaluating the set of first and second switching points.

5. Conclusion. In this article we have classified the optimal syntheses for the time minimal control problem, with terminal manifold of codimension one $N$, near $N$ and under generic assumptions, for scalar affine system (1), when $v \in \mathbf{R}^{2}$ or $\mathbf{R}^{3}$. Our study is mainly topological. In a forthcoming article we shall complete 
our study in the following directions: give linear estimates of the strata of $K \cup C$ and more generally find an algorithm to compute all stratum up to a required precision. In the control of chemical batch reactor [3] the situation is not generic because $Y$ is everywhere tangent to $N$, and we have analyzed this situation. Due to space restriction, this analysis cannot be presented here and will be published later. Of course the ultimate goal of such a study is to compute the stratification associated to a singularity theory of the time minimum function $v \rightarrow T(v)$.

\section{References}

[1] R. Benedetti and J. J. Risler, Real Algebraic and Semi-Algebraic Sets, Hermann, Paris, 1990.

[2] B. Bonnard et I. Kupka, Théorie des singularités de l'application entrée/sortie et optimalité des trajectoires singulières dans le problème du temps minimal, Forum Math. 5 (1993), 111-159.

[3] B. Bonnard and J. de Morant, Towards a geometric theory in the time minimal control of chemical batch reactors, to appear in SIAM J. Control Optim.

[4] B. Bonnard and M. Pelletier, Time minimal synthesis for planar systems in the neighborhood of a terminal manifold of codimension one, preprint, Laboratoire de Topologie de Dijon, 1992, to appear in JMSEC.

[5] I. Kupka, Geometric theory of extremals in optimal control problems. I. The fold and Maxwell cases, Trans. Amer. Math. Soc. 299 (1977), 225-243.

[6] E. B. Lee and L. Markus, Foundations of Optimal Control Theory, Wiley, New York, 1967.

[7] H. Poincaré, Sur les lignes géodésiques des surfaces convexes, Trans. Amer. Math. Soc. 6 (1905), 237-274.

[8] H. Schättler, The local structure of time optimal trajectories under generic conditions, SIAM J. Control Optim. 26 (1988), 899-918.

[9] H. J. Sussmann, The structure of time optimal trajectories for single-input systems in the plane: the $C^{\infty}$ non singular case, ibid. 25 (1987), 433-465.

[10] - Regular synthesis for time optimal control single-input real analytic systems in the plane, ibid. 25 (1987), 1145-1162. 